ESAIM: PROCEEDINGS, May 2009, Vol. 27, p. 90-106

C. Besse, O. Goubet, T. Goudon \& S. Nicaise, Editors

\title{
MODÉLISATION DES DÉPRESSIONS CORTICALES ENVAHISSANTES ET EXISTENCE DE FRONTS PROGRESSIFS
}

\author{
Guillemette Chapuisat ${ }^{1}$
}

Résumé. Lors d'un accident vasculaire cérébral ou d'une migraine avec aura, des ondes de dépolarisation se propagent dans la substance grise du cerveau du rat. Le blocage de ces ondes est une cible thérapeutique importante, mais l'existence de ces ondes dans le cerveau humain reste à prouver. Les mécanismes sont pourtant identiques chez les deux espèces, ce sont des mécanismes de type réaction-diffusion. Notre hypothèse est que la morphologie du cerveau humain pourrait expliquer les difficultés d'observation de ces ondes, car elle limiterait leur propagation sur de longues distances.

Après une courte introduction biologique, on explique la construction du modèle puis on présente différentes expériences numériques qui portent sur l'influence de la morphologie du cerveau sur la propagation des ondes de dépolarisation.

On étudie ensuite plus en détails l'équation suivante :

$$
\partial_{t} u-\triangle u=\lambda u(u-\theta)(1-u) 1_{|z|<R}-\alpha u 1_{|z| \geq R}
$$

où $(x, z) \in \mathbb{R}^{N}, x \in \mathbb{R}$ et $\lambda u(u-\theta)(1-u)$ est la non-linéarité bistable usuelle. Il s'agit donc d'une équation de réaction-diffusion sur un cylindre de rayon $R$ couplée avec une équation de diffusion et absoption en dehors du cylindre. On démontre que pour $R$ petit, il n'existe pas de front progressif solution de cette équation, mais que pour $R$ grand, il existe un tel front. Pour cela, on étudie les différents profils asymptotiques possibles pour le front. Cela suffit à montrer la non-existence de front si $R$ est petit. Ensuite pour construire un front progressif quand $R$ est assez grand, on étudie l'énergie d'une solution avec une donnée initiale bien choisie dans différents référentiels en mouvement.

\begin{abstract}
Spreading depression is a transient depolarization of neurons that spreads slowly through a part of the brain during stroke, epilepsy or migraine with aura. They have been observed and studied in most animal species for more than 50 years, but their existence in the human brain is still discussed. Mathematical models of spreading depressions have been established; they are linked to a reaction-diffusion mechanism. After some numerical experiments, we have made the following hypothesis: The mecanisms that trigger spreading depressions are the same in the human brain as in the rodent brain for example, but the morphology of the human brain could explain the non-observation of these waves. Hence we have studied the following equation:$$
\partial_{t} u-\triangle u=\lambda u(u-\theta)(1-u) 1_{|z|<R}-\alpha u 1_{|z| \geq R}
$$

where $(x, z) \in \mathbb{R}^{N}$ is the space variable. We have proved that if $R$ is small enough, there is no travelling front solution of these equation. And if $R$ is large enough, there exists a travelling front in the $x$-direction. This result is obtained by studying the energy of a solution with special initial conditions in several travelling referentials.
\end{abstract}

\footnotetext{
1 CMLA, ENS Cachan, CNRS, Pres UniverSud, 94235 Cachan, France
} 


\section{INTRODUCTION}

La modélisation mathématique en biologie ou en médecine est un domaine en plein essor. Grâce à la modélisation, certains traitements médicaux ont pu être adaptés et améliorés. Inversement, la complexité des phénomènes biologiques a amené de nouvelles avancées dans le domaine du calcul scientifique, des équations aux dérivées partielles ou des statistiques par exemple.

Dans cet article, j'aborde le problème de l'existence des dépressions corticales envahissantes dans le cerveau humain. Ces dépressions corticales ont un rôle majeur dans la lutte contre les accidents vasculaires cérébraux, mais aussi pour le traitement des migraines avec aura. C'est un exemple où la modélisation mathématique a permis d'émettre de nouvelles hypothèses biologiques et où les questions des médecins ont été la source de nouveaux problèmes théoriques d'existence de front progressifs.

La première partie de ce travail est donc consacrée à la description du modèle de dépression corticale envahissante après une courte introduction biologique. On présente ensuite les différentes simulations numériques réalisées dans le but de répondre aux questions des médecins. La seconde partie est plus théorique. Elle s'intéresse à un problème d'existence de front progressif issu de la modélisation.

\section{Un MOdÈLE PHÉNOMÈnOLOGIQUE DE DÉPRESSION CORTICALE ENVAHISSANTE}

\subsection{Les dépressions corticales envahissantes}

Avant de pouvoir définir les dépressions corticales envahissantes, il est nécessaire de savoir que le cerveau est composé de différentes cellules. Nous nous intéresserons ici aux neurones dont le rôle est de transmettre l'influx nerveux et aux astrocytes dont le rôle est de maintenir un environnement favorable au bon fonctionnement des neurones. Pour pouvoir transmettre les messages nerveux, la membrane des neurones doit être polarisée, c'est à dire que les ions sont répartis de façon spécifique entre l'intérieur et l'extérieur de la cellule par un système de pompes ioniques.

Une dépression corticale envahissante (DCE), ou spreading depression (SD) en anglais, est une dépolarisation temporaire de grande ampleur des neurones qui se propage lentement $(3 \mathrm{~mm} / \mathrm{min})$ dans la substance grise du cerveau. Ce phénomène a été observé en premier par Leão en 1957 [18] et est depuis le sujet de nombreuses études. Ces DCE semblent jouer un rôle important dans diverses pathologies, en particulier les accidents vaculaires cérébraux (AVC), les migraines avec aura, l'épilepsie et certains traumatismes crâniens [32].

La mesure des DCE est assez difficile. Un électroencéphalogramme ne suffit pas [17] et même l'IRM ne permet pas de conclure de manière définitive [15]. Les seuls moyens de mesure efficaces sont invasifs.

Dans le cadre d'un AVC chez le rongeur, le nombre de DCE a une claire influence sur la taille de la zone définitivement lésée [21,23]. Certains essais thérapeutiques portent sur des bloqueurs de ces DCE et donnent de bons résultats chez le rongeur. Malheureusement la situation chez l'homme est moins claire, car les observations sont bien plus difficiles à obtenir. En réalité, la question de l'existence de ces DCE chez l'homme n'est toujours pas résolue. Diverses opinions s'affrontent [1,19,20,33]. Pour l'instant aucune observation claire de ces DCE chez l'homme n'a été faite. Ce point est pourtant essentiel, car cela pourrait réorienter certains essais thérapeutiques.

La quantité importante de connaissances disponibles à propos des DCE et les difficultés d'expérimentation en particulier chez l'être humain ont mené les biologistes à créer des modèles mathématiques de DCE. Dans [32], G. Somjen expose un modèle de DCE qui s'intéresse particulièrement au déclenchement et à l'évolution de la DCE dans une cellule unique dans le cas de l'épilepsie et de l'AVC ischémique. Il est composé d'équations différentielles ordinaires. Malheureusement, ce modèle ne peut pas prendre en compte la propagation des DCE dans le cerveau qui est pourtant 


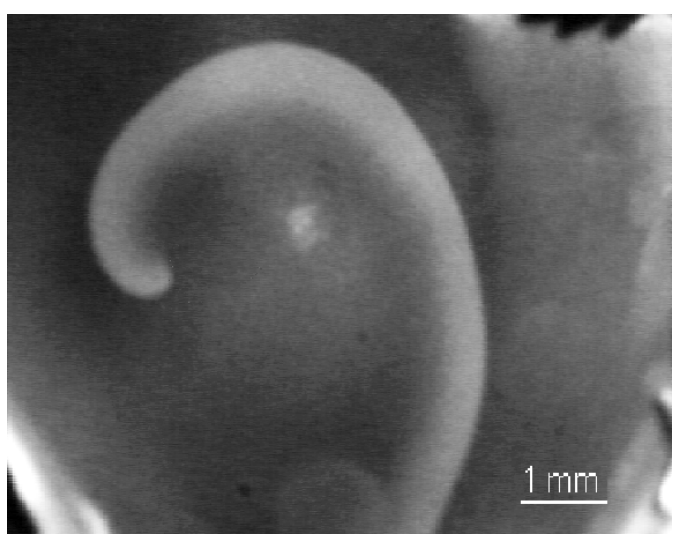

Figure 1. Une dépression corticale envahissante sur la rétine d'un poulet déclenchée par une injection de $\mathrm{KCl}$. Les neurones qui composent la rétine ont la particularité d'être opaques quand ils sont dépolarisés.

probablement une des raisons de l'extension des dommages dans le cadre des AVC. D'autres modèles utilisant des équations de réaction-diffusion ont été développés dans $[14,22,26,27,34]$. Ces modèles plus simples biologiquement sont en revanche centrés sur le problème de la propagation des DCE et ne sont que sommairement rattachés aux observations physico-biologiques au niveau membranaire. Par exemple, ils ne permettent pas d'expliquer les observations de Nedergaard [5] sur la présence d'une vague de calcium dans les astrocytes. Enfin, Shapiro dans [30] présente un modèle très complet de DCE qui suit quasiment toutes les concentrations ioniques en jeu dans la cellule, mais ce modèle est très lourd et pourrait difficilement être inclus dans un modèle global d'AVC. Nous avons donc choisi d'établir un nouveau modèle phénomènologique de DCE qui ne représenterait que quelques espèces ioniques et qui pourrait ensuite être inclus dans un modèle complet d'AVC comme cela a été fait dans [7]. Ce modèle de DCE a été proposé pour la première fois dans [8]. Et dans [7], on revient plus en détails sur la biologie de ce modèle.

\subsection{Les hypothèses biologiques du modèle}

Les DCE étant le fait d'échanges ioniques anormaux entre les neurones, les astrocytes et l'espace extracellulaire, ce modèle de DCE considère trois compartiments différents : les neurones, les astrocytes et l'espace extracellulaire. Cependant, on se place à l'échelle du cerveau, les grandeurs varient donc continûment dans ces trois compartiments.

Les concentrations en potassium et en calcium jouent des rôles essentiels dans le phénomène de DCE. Les principales fonctions de l'espace $x$ et du temps $t$ de ce modèle sont donc : $K_{e}(t, x)$ la concentration en potassium dans l'espace extracellulaire, $C a_{n}(t, x)$ la concentration en calcium dans les neurones et $C a_{g}(t, x)$ la concentration en calcium dans les astrocytes ( $g$ pour gliale).

Le modèle de DCE a été fondé principalement sur les observations de Nedergaard dans [5]. Les principaux faits sont les suivants :

- Les ions potassium peuvent diffuser dans l'espace extracellulaire.

- Un excès de potassium dans l'espace extracellulaire ouvre des canaux ioniques sur la membrane des astrocytes qui font entrer du calcium dans la cellule.

- Le calcium diffuse à travers des gap-jonctions dans le réseau des astrocytes.

- Les ions calcium entrent dans les neurones "à travers des gap-jonctions entre neurones et astrocytes".

- L'excès de calcium dans les neurones ouvre des canaux ioniques sur la membrane des neurones qui font sortir du potassium de la cellule. 
Ces points sont résumés sur la figure 2 .

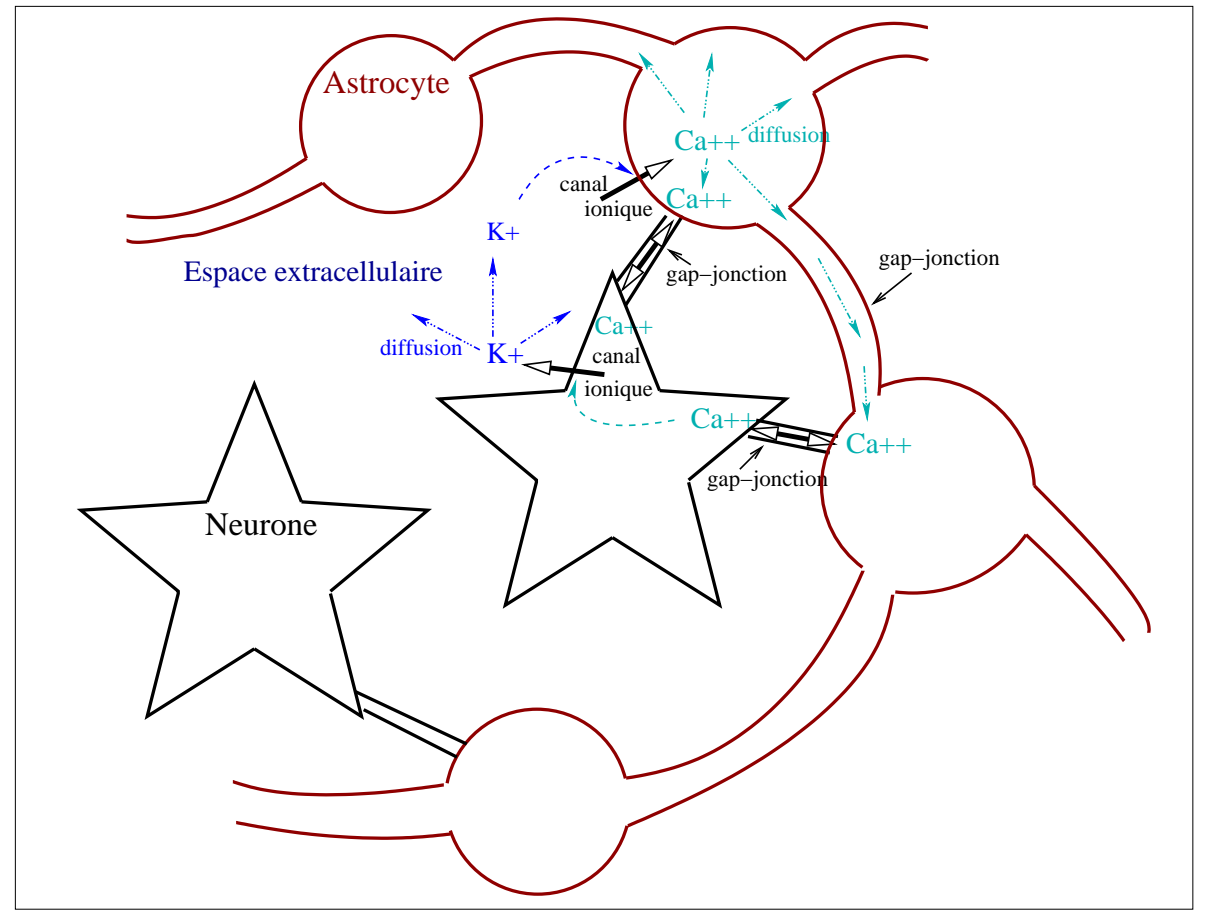

Figure 2. Le modèle de Nedergaard

Nous n'avons parlé pour l'instant que d'augmentations des concentrations ioniques, il est donc nécessaire d'ajouter des phénomènes de récupération. Les connaissances biologiques sur ces phénomènes sont limitées. On sait cependant qu'il existe différents mécanismes de retour à l'équilibre, pour les petites variations de concentrations (induites par le passage d'un signal nerveux par exemple) ou pour récupérer des larges variations de concentrations produites par une DCE.

La récupération des DCE nécessite deux nouvelles inconnues : $C a p(t, x)$ la capacité des cellules à récupérer des $\mathrm{DCE}$ et $R(t, x)$ l'intensité de cette récupération.

On obtient finalement un système de trois équations aux dérivées partielles de réaction-diffusion pour les concentrations ioniques et de deux équations différentielles ordinaires (mais où la variable d'espace intervient comme paramètre) pour gérer la récupération des DCE. 


$$
\begin{aligned}
\partial_{t} C a_{g}= & \nu_{C a_{g}} \Delta C a_{g}-k_{C a_{g}} \tanh \frac{C a_{g}}{C a_{g}^{0}}+h_{K, C a} K_{e}-k_{g a p}\left(C_{a_{g}}-C_{a_{n}}\right) \\
& -\alpha_{C a_{g}} C a_{g}^{2}-\alpha_{r e c, g} R C a_{g}
\end{aligned}
$$

Pour une explication détaillée des différents termes de ces équations, je renvoie aux annexes de [7].

\subsection{Résultats sur un cerveau "carré"}

Pour déterminer les valeurs des différents paramètres, on a utilisé un algorithme aléatoire décrit en détails dans [8]. On trouve 6 jeux différents de paramètres et après les avoir testés dans différentes situations, on constate que les résultats sont qualitativement identiques pour les différents jeux de paramètres trouvés. Par exemple, la figure 3 montre le comportement du système quand on simule un AVC en deux dimensions d'espace sur un morceau cerveau carré. Le système d'équations est résolu à l'aide d'une transformée de Fourier rapide en espace et d'un schéma semi-implicite en temps.

\subsection{Le rôle de la géométrie du cerveau}

\subsubsection{Composition et géométrie du cerveau}

Le cerveau est composé de deux substances différentes.

- La substance grise, principalement composée des corps neuronaux et d'astrocytes, constitue donc le siège de l'activité cérébrale.

- La substance blanche, elle, contient principalement les axones neuronaux, des astrocytes et d'autres cellules dites gliales. Elle assure donc surtout la conduction de l'influx nerveux.

Selon l'espèce, la composition et la géométrie du cerveau peut être très variable. Le cerveau du rongeur par exemple est quasiment entièrement composé de substance grise. De plus, il présente très peu de replis. Le cerveau de l'homme en revanche est très différent. Il est divisé en deux hémisphères. Chaque hémisphère est divisé en quatre lobes, divisés eux-même en de nombreuses sous-parties appelées circonvolutions, séparées par des sillons ou scissures. De plus, il est principalement composé de substance blanche, la substance grise étant surtout présente au niveau du cortex cérébral qui compose une fine couche à la superficie du cerveau. En raison des circonvolutions et des scissures, cette couche de substance grise peut être d'épaisseur très variable. Le modèle de DCE présenté cidessus n'est valide que dans la substance grise. Dans la substance blanche, il n'y a plus de neurones, donc il n'y a plus de "gap-jonctions entre les neurones et les astrocytes". Le paramètre $k_{g} a p$ du système d'équation est donc ramené à 0 . De même pour $h_{C a, K}$ et la concentration en calcium dans les neurones restera donc à 0 dans la substance blanche. Le reste du système d'équations est gardé, la diffusion est inchangée (mais il n'y a plus de réaction). 

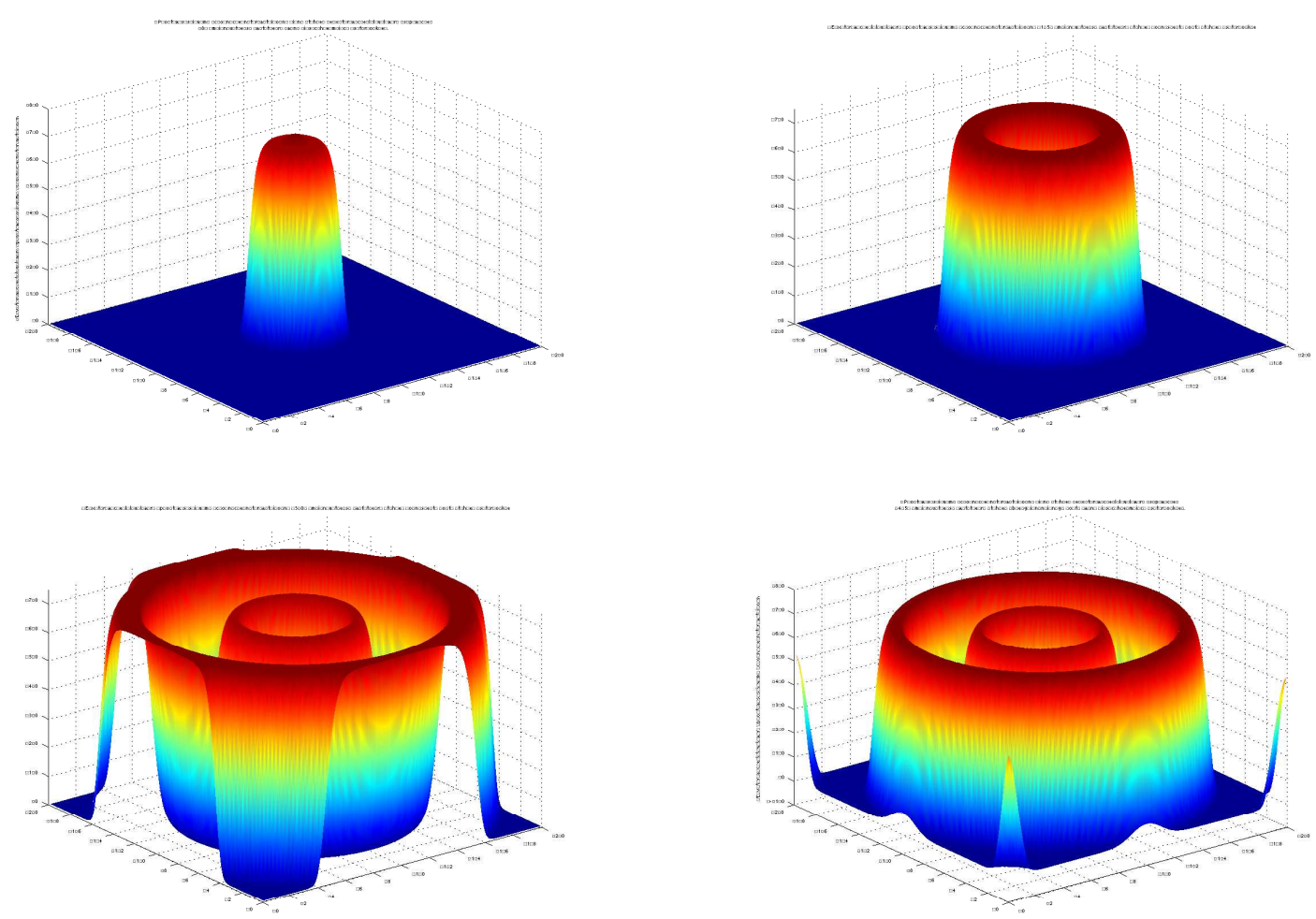

Figure 3. Concentration en potassium dans un cerveau carré à la suite d'un AVC $(5,15,30$ et 45 min après le début de l'AVC). Plusieurs DCE se propagent.

\subsubsection{Effet d'une variation d'épaisseur de la substance grise}

On s'intéresse ici à l'influence de la géométrie de la substance grise sur la propagation des DCE. Nous avons voulu voir si les variations d'épaisseur de la substance grise peuvent être un obstacle à la propagation des DCE. On observe qu'une diminution de l'épaisseur de la substance grise n'affecte pas la propagation des DCE. En revanche, une augmentation brutale d'épaisseur de la substance grise empêche la propagation des DCE (voir figure 6). Ces résultats ont été détaillés dans [8]

Ces résultats peuvent être interprétés biologiquement : la création des DCE se ferait de même chez l'homme et chez le rongeur, mais dans le cerveau humain, la propagation de ces ondes s'arrêterait à la première augmentation brutale de l'épaisseur de la substance grise, ce qui rendrait les ondes difficiles à observer.

La démonstration théorique de l'extinction des ondes dans un cylindre dont le diamètre augmente brutalement a été faite pour une équation plus simple que le système issu de la modélisation. On peut trouver ce résultat dans [9].

\subsubsection{Effet de la substance blanche}

On a également étudié numériquement l'influence de la substance blanche sur la création de DCE. En effet, comme la substance blanche ne contient pas de neurone, les ions responsables des DCE y diffusent simplement et sont progressivement absorbés. L'étape de réaction n'a lieu que dans la substance grise. L'effet absorbant de la substance blanche pourrait donc empêcher la création de DCE. Or le cerveau du rat contient très peu de substance blanche, alors que le cerveau humain est principalement composé de cette substance avec une fine couche de substance grise à la périphérie. 

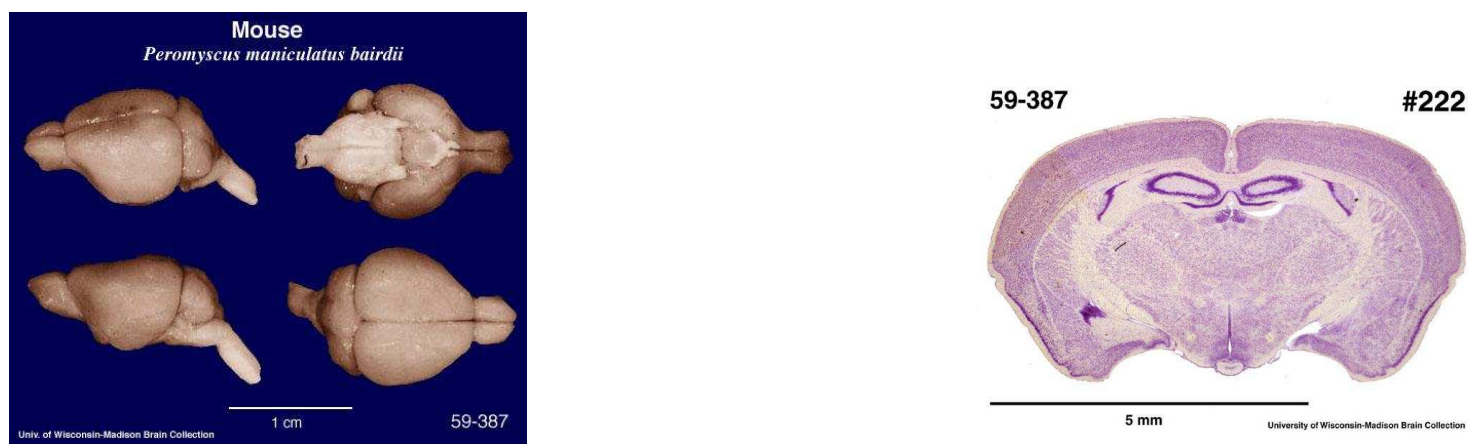

Figure 4. Le cerveau de la souris. A gauche, différentes vues. A droite, une coupe. (http ://www.brainmuseum.org/index.html)
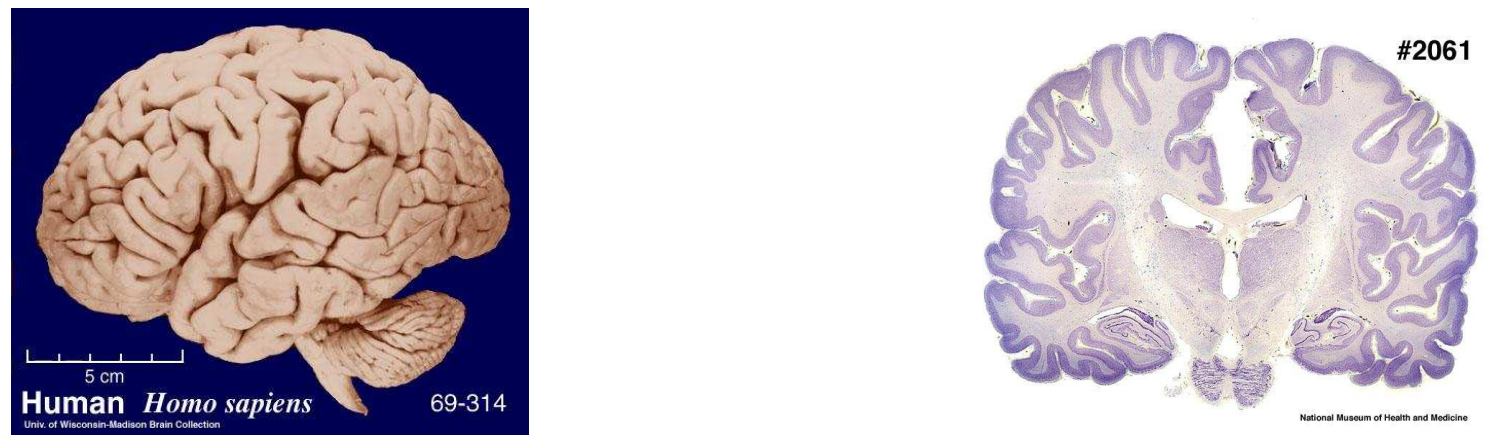

Figure 5. Le cerveau de l'homme. A gauche, une vue d'ensemble. A droite, une coupe. (http ://www.brainmuseum.org/index.html)

Avec ce modèle de DCE, on constate effectivement que si l'épaisseur de la substance grise est trop faible, alors une simulation d'injection de potassium ne crée plus de DCE (cf figures 7 et 8).Ces résultats ont été détaillés dans [8]

Ces résultats signifient que même si le mécanisme de création des ondes est le même chez l'homme et chez le rongeur, la finesse de la substance grise dans le cerveau humain pourrait empêcher la création de DCE dans certaines zones.

La démonstration théorique de ce résultat pour le système complet issu de la modélisation est difficile. La partie suivante porte sur ce problème d'existence de front mais pour une simple équation scalaire reprenant les principales caractéristiques de ce modèle.

\section{Existence DE Fronts PROGRESSIFS COURBES}

Dans cette partie, j'étudie les fronts progressifs solutions d'une équation de réaction-diffusion sur un cylindre de rayon $R$ dans $\mathbb{R}^{N}$ et d'une équation de diffusion et absorption en dehors du cylindre.

\subsection{Enoncé du théorème}

Je m’intéresse à l'équation suivante :

$$
\frac{\partial u}{\partial t}-\triangle u=g(u) 1_{\Omega}-\alpha u 1_{\mathbb{R}^{N} \backslash \Omega}, \quad t \in \mathbb{R}, X \in \mathbb{R}^{N}
$$



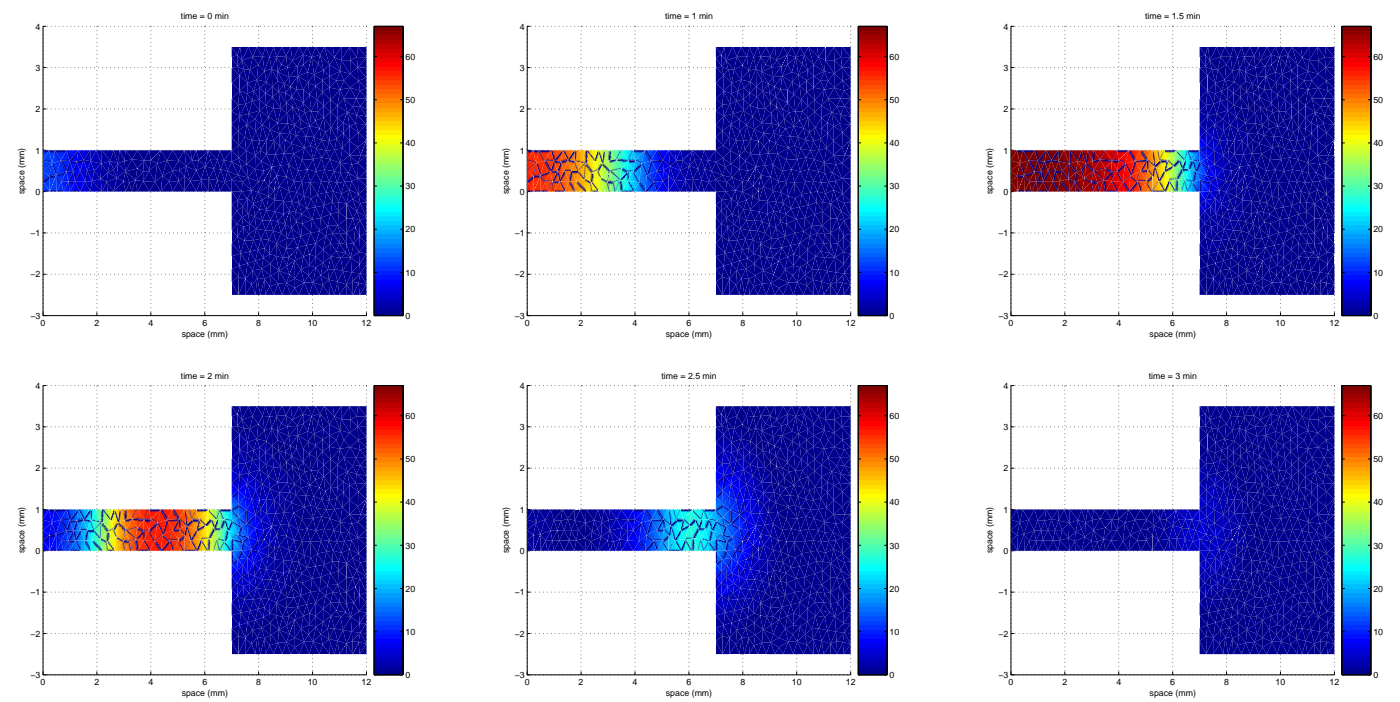

Figure 6. Concentration en potassium dans l'espace extracellulaire à différents temps. Ici, la substance grise est représentée par un cylindre de diamètre 1 puis 6. Les DCE sont arrêtées par l'augmentation brutale de l'épaisseur de la substance grise.

où $g(u)=\lambda u(u-\theta)(1-u)$ est la nonlinéarité bistable usuelle avec $\theta \in] 0,1 / 2[, 1$ désigne une fonction caractéristique d'un ensemble et $\Omega$ est un cylindre infini de rayon $R$ représentant la substance grise (réaction-diffusion).

Dans la suite, on notera la variable d'espace $(x, z) \in \mathbb{R}^{N}$ avec $x \in \mathbb{R}$ et $z \in \mathbb{R}^{N-1}$, et $\Omega=$ $\mathbb{R} \times B(0, R)$ le cylindre.

On cherche les solutions de (6) de type front progressif courbe, c'est à dire $u(t, x, z)=U(x-$ $c t, z)$ pour un $c \in \mathbb{R}$ constant à déterminer, avec de plus les limites uniformes $\lim _{x \rightarrow-\infty} U(x, z)$ et $\lim _{x \rightarrow+\infty} U(x, z)$ qui existent et sont distinctes. Le profil de l'onde $U$ vérifie donc l'équation

$$
(\mathrm{TF}) \begin{cases}\triangle U+c \partial_{x} U+g(U) 1_{\{|z| \leq R\}}-\alpha U 1_{\{|z|>R\}}=0 & (x, z) \in \mathbb{R}^{N} \\ U(x, z) \rightarrow 0 \text { pour }|z| \rightarrow \infty, & x \in \mathbb{R}\end{cases}
$$

et les profils asymptotiques $\phi_{ \pm}=\lim _{x \rightarrow \pm \infty} U$ sont solutions de l'équation

$$
\left(P_{\infty}\right)\left\{\begin{array}{l}
\triangle_{z} \phi+g(\phi) 1_{\{|z| \leq R\}}-\alpha \phi 1_{\{|z|>R\}}=0, \quad z \in \mathbb{R}^{N-1} \\
\phi(z) \underset{|z| \rightarrow \infty}{\longrightarrow} 0 .
\end{array}\right.
$$

Dans [6], j'ai montré les théorèmes suivants.

Théorème 2.1. Si $N=2$, il existe $R_{0}>0$ tel que

- si $R<R_{0}, \phi \equiv 0$ est la seule solution $C^{1}$ de $\left(P_{\infty}\right)$,

- si $R>R_{0},\left(P_{\infty}\right)$ admet au moins 3 solutions $C^{1}: 0, \phi_{1}>0$ et $\phi_{2}>0$.

Théorème 2.2. Si $N \geq 3$, il existe $R_{1} \geq R_{0}>0$ tel que

- si $R<R_{0}, \phi \equiv 0$ est la seule solution radiale $C^{1}$ de $\left(P_{\infty}\right)$,

- si $R>R_{1},\left(P_{\infty}\right)$ admet au moins 2 solutions $C^{1}$ : 0 et $\phi>0$. 

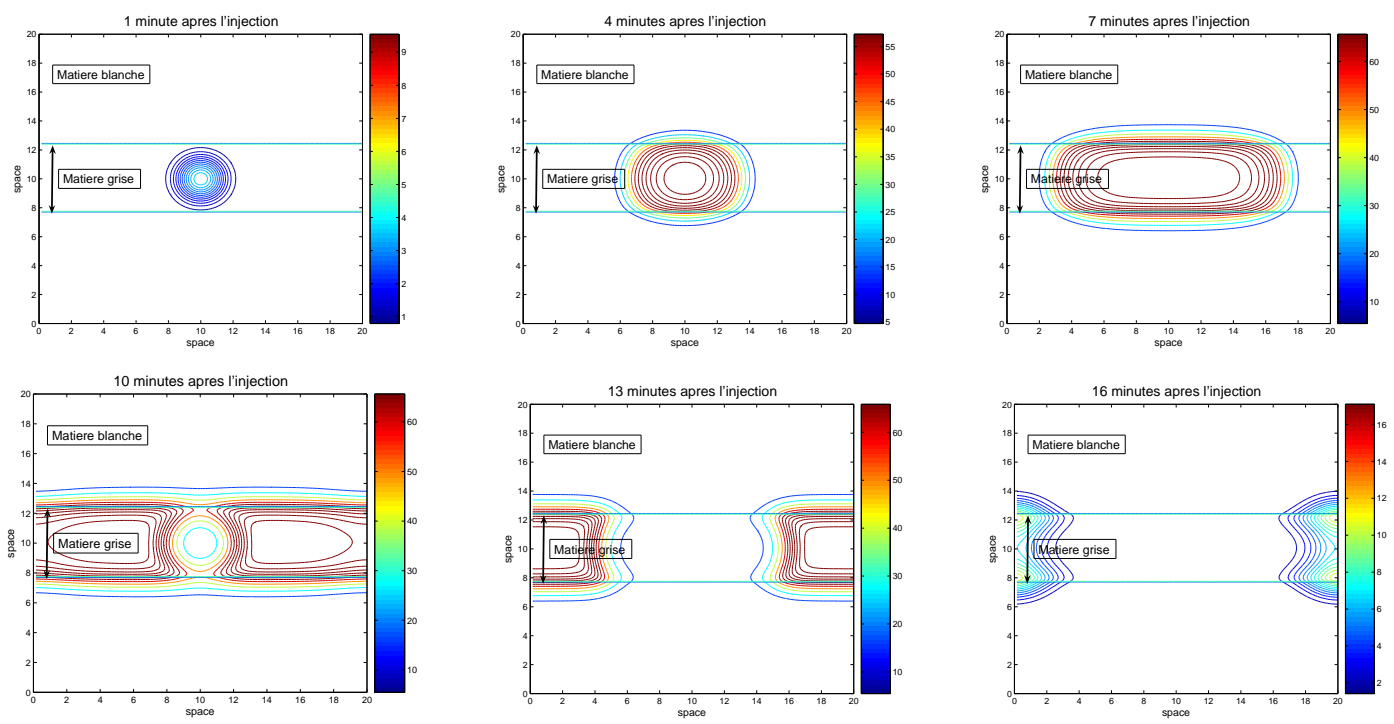

Figure 7. Concentration en potassium dans l'espace extracellulaire après une injection de $\mathrm{KCl}$. La couche de substance grise est épaisse. Une DCE se crée.
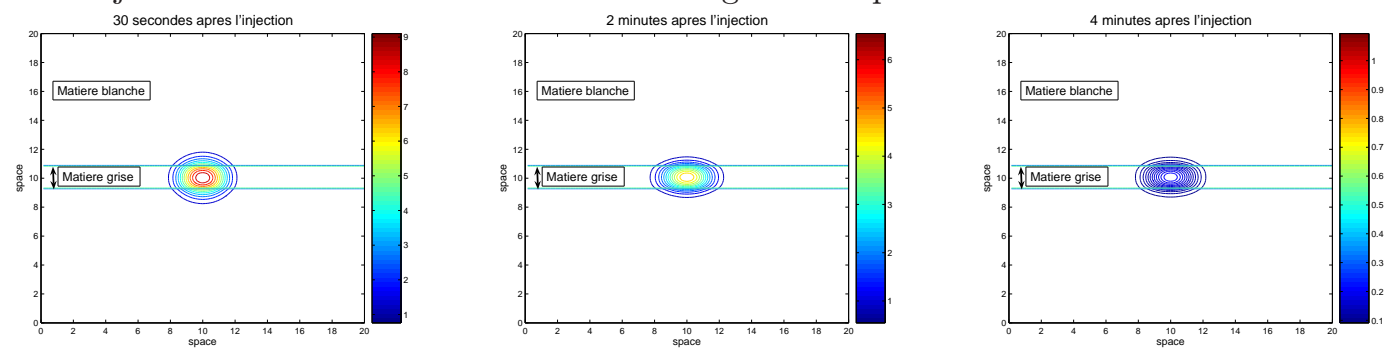

Figure 8. Concentration en potassium dans l'espace extracellulaire après une injection de $\mathrm{KCl}$. La couche de substance grise est fine. Aucune DCE n'est créée.

Corollaire 2.3. Si $N=2$ (resp. $N \geq 3$ ), il n'existe pas de front progressif (resp. de front progressif cylindrique) solution de (6) pour $R<R_{0}$.

Si $R \geq R_{0}$, l'existence de front d'onde et la convergence des solutions vers ces fronts d'onde est le point le plus délicat à montrer. Historiquement, l'étude des fronts d'onde en dimension 1 d'espace a commencé avec Aronson et Weinberger [2]. Ils ont montré l'existence de front en travaillant dans le plan de phase. Puis Fife et McLeod $[11,12]$ ont fait une démonstration de la convergence des solutions vers ces fronts en utilisant principalement le principe du maximum et en construisant des sur- et sous-solutions bien adaptées.

En dimension supérieure, il existe plusieurs types de résultats. Berestycki et Nirenberg [3] ont montré l'existence de fronts progressifs dans des cylindres avec un terme d'entrainement qui peut dépendre des coordonnées transverses. Vega [35] a montré l'existence et l'unicité de front progresifs dans un cylindre avec des conditions aux bords de Dirichlet homogènes. Enfin, Roquejoffre a étudié la convergence vers des fronts sur un cylindre [29]. Tous ces articles utilisent de façon plus ou moins précise l'énergie de la solution et le linéarisé à l'infini. Ils ont donc tous besoin que le cylindre soit de section bornée pour pouvoir utiliser des injections de Sobolev compactes, mais aussi des fonctions propres du linéarisé.

Sur l'espace entier, Hamel, Monneau et Roquejoffre ont étudié le problème de la combustion de la 
flamme du bec Bunsen [16]. Leur démonstration utilise de nombreuses fois la méthode de glissement introduite dans [4] par Berestycki et Nirenberg ainsi que l'existence d'un front en dimension 1 d'espace. En effet, leur front est un front conique, donc il ressemble à un front plan à l'infini (donc à un front en dimension 1 d'espace). En particulier, ils connaissent la vitesse du front a priori et peuvent ainsi construire des sur- et sous-solution adaptées.

L'autre approche de l'étude des fronts progressifs vient des problèmes de cinétique chimique. Dans ce cas, les fronts sont vectoriels et il n'existe plus de principe du maximum. Une possibilité est d'utiliser la méthode de Leray-Schauder comme Volpert [36] ou bien Gardner [13]. Une autre méthode a été développée par Emmanuel Risler [28]. Elle montre l'existence de fronts progressifs et un début de convergence en dimension 1 d'espace en étudiant l'énergie des solutions dans différents référentiels en mouvement. Cette méthode a été développée pour éviter l'utilisation du principe du maximum qui existe dans notre cas, mais elle présente également de nombreux autres intérêts. Tout d'abord, la méthode détermine directement la vitesse du front et ne demande pas d'en connaître un encadrement a priori. Ensuite, cette méthode utilise l'énergie des solutions, donc des intégrales. La taille de la section du cylindre n'a donc pas d'importance, il suffit que la solution soit intégrable en $z$ à $t$ et $x$ fixés.

\subsection{La preuve du théorème}

\subsubsection{Etude des profils asymptotiques}

Avant de montrer l'eventuelle existence de front progressif, il est naturel de chercher à connaître les états que pourrait relier ce front, c'est à dire les différents profils asymptotiques possibles. Rappelons qu'un tel profil asymptotique est solution du problème

$$
\left(P_{\infty}\right)\left\{\begin{array}{l}
\triangle \phi+g(\phi) 1_{\{|z| \leq R\}}-\alpha \phi 1_{\{|z|>R\}}=0 \quad z \in \mathbb{R}^{N-1} \\
\phi(z) \underset{|z| \rightarrow \infty}{\longrightarrow} 0 .
\end{array}\right.
$$

En dimension $N=2$, cette équation devient une équation différentielle ordinaire et on peut travailler dans le plan de phase.

\subsubsection{Cas de la dimension $N=2$}

Cette partie donne les idées de la démonstration du théoreme 2.1. Tout d'abord, introduisons quelques notations. On définit

$$
\left(E_{1}\right) \quad \phi^{\prime \prime}+g(\phi)=0 \quad \text { et } \quad\left(E_{2}\right) \quad \phi^{\prime \prime}-\alpha \phi=0
$$

Le problème est de recoller une solution de $\left(E_{1}\right)$ avec une solution de $\left(E_{2}\right)$ de façon $C^{1}$ en $z=-R$ et en $z=R$. Les solutions de $\left(E_{2}\right)$ qui tendent vers 0 en l'infini sont bien connues. On a donc $\phi(z)=B e^{\sqrt{\alpha} z}$ pour un $B \in \mathbb{R}$ si $z<-R$, et $\phi(z)=A e^{-\sqrt{\alpha} z}$ pour un $A \in \mathbb{R}$ si $z>R$. Donc le problème est de trouver une solution de $\left(E_{1}\right)$ telle que

$$
\left(B C_{1}\right) \quad \phi^{\prime}(-R)=\sqrt{\alpha} \phi(-R) \text { et } \phi^{\prime}(R)=-\sqrt{\alpha} \phi(R)
$$

En travaillant dans le plan de phase (cf figure 9), on peut étudier le temps que met une trajectoire de $\left(E_{1}\right)$ passant par $\left(\phi_{c},-\sqrt{\alpha} \phi_{c}\right)$ pour joindre les deux droites $\phi^{\prime}=\sqrt{\alpha} \phi$ et $\phi^{\prime}=-\sqrt{\alpha} \phi$. Par continuité du flot, on voit assez facilement que ce temps est défini et continu en fonction de $\phi_{c}$ sur un intervalle $\left[0, \phi_{\max }\right]$ et qu'en chaque extrémité de cet intervalle, ce temps tend vers $+\infty$ car la trajectoire devrait passer par un équilibre de $\left(E_{1}\right)$ (0 ou 1). Ainsi, il existe un temps minimum $R_{0}>0$ pour qu'une trajectoire de $\left(E_{1}\right)$ joigne les deux trajectoires voulues de $\left(E_{2}\right)$, on en déduit le théorème 2.1 . 

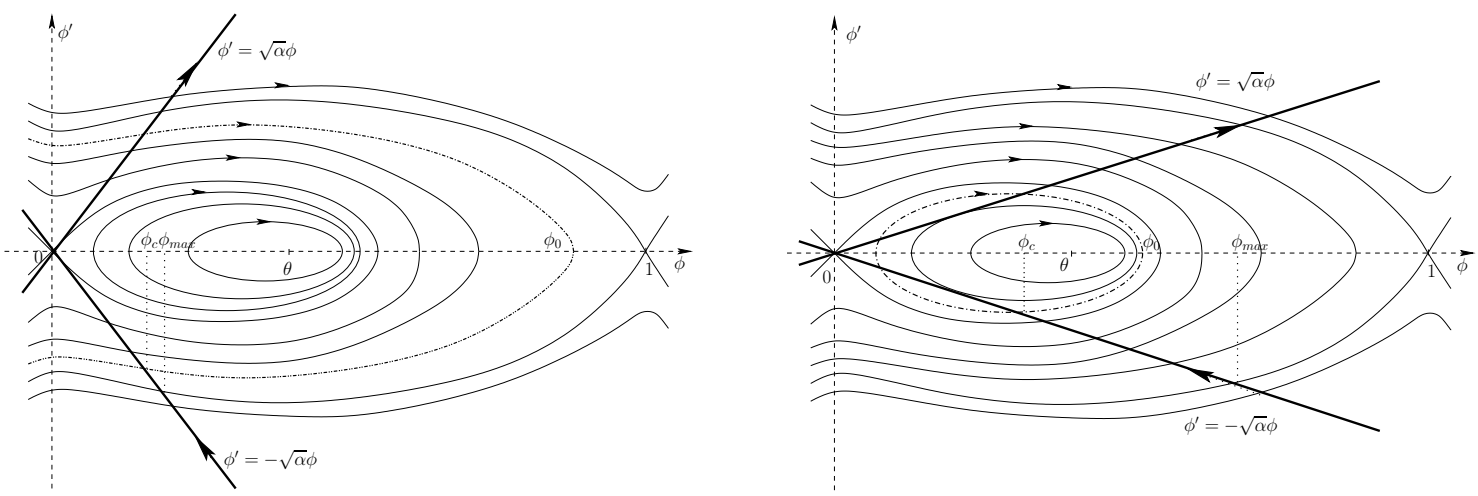

Figure 9. Superposition du plan de phase de $\left(E_{1}\right)$ et des trajectoires de $\left(E_{2}\right)$ qui nous intéressent ici. A gauche, dans le cas $\sqrt{\alpha} \geq \theta$ et à droite pour $\sqrt{\alpha}<\theta$.

\subsubsection{Cas de la dimension $N>2$}

En dimension supérieure, si on suppose les solutions radiales, les profils asymptotiques vérifient l'équation

$$
\text { (ode) }\left\{\begin{array}{l}
\psi^{\prime \prime}+\frac{N-2}{r} \psi^{\prime}+g(\psi) 1_{\{r \leq R\}}-\alpha \psi 1_{\{r>R\}}=0 \\
\psi(r) \underset{r \rightarrow \infty}{\longrightarrow} 0, \quad \psi^{\prime}(0)=0 .
\end{array}\right.
$$

J'ai montré le théorème 2.2 en utilisant les solutions construites en dimension $N=2$.

Supposons par l'absurde qu'il existe une solution $\psi$ non identiquement nulle de (ode) pour $R<R_{0}$. Alors, si $\psi$ est décroissante, $\psi$ est clairement une sous-solution de $\left(P_{\infty}\right)$ en dimension 1 . 1 étant une sursolution, on peut construire une solution de $\left(P_{\infty}\right)$ en dimension 1 comprise entre $\psi>0$ et 1 (cf [10] ou [31] par exemple). Cela contredirait le théorème 2.1. Maintenant, $\psi$ n'est pas nécessairement décroissante, mais par le principe du maximum, comme $\psi$ tend vers 0 en l'infini, il existe $M>0$ tel que $\psi$ est décroissante sur $\left[M,+\infty\left[\right.\right.$ et $\psi^{\prime}(M)=0$. On peut alors construire une sous-solution non nulle de $\left(P_{\infty}\right)$ en dimension 1 en posant $\phi(z)=\psi(M+|z|)$. Ceci contredit le théorème 2.1, il n'existe donc pas de solution de (ode) pour $R<R_{0}$.

Pour trouver une solution de (ode) si $R$ est suffisament grand, on va construire une sous-solution strictement positive. Pour cela, on constate que pour $\phi$ la plus grande des solutions de $\left(P_{\infty}\right)$ construites en dimension 1 , le terme $\frac{N-2}{r} \phi^{\prime}$ peut être aussi petit que souhaité si $R$ est suffisament grand. On peut alors construire une nouvelle nonlinéarité $g_{0}$ et un coefficient $\alpha_{0}$ qui vérifient les hypothèses du théorème 2.1 et tels que

$$
\frac{N-2}{r} \phi^{\prime}+\left(g(\phi)-g_{0}(\phi)\right) 1_{\{r \leq R\}}-\left(\alpha-\alpha_{0}\right) \phi 1_{\{r>R\}} \geq 0 .
$$

La plus grande des solutions de l'équation avec $g_{0}$ et $\alpha_{0}$ en dimension 1 est donc une sous-solution strictement positive de (ode). On en déduit donc l'existence d'une solution. Tous les calculs sont détaillés dans [6].

La non existence de profil asymptotique autre que 0 si $R<R_{0}$ entraîne la non existence de front progressif solution de $(T F)$ si $R<R_{0}$. Il reste à montrer l'existence d'un front progressif lorsqu'il existe différents profils asymptotiques. On supposera donc désormais $R>R_{1}$.

\subsubsection{Idées de la preuve de l'existence de fronts}

Pour montrer l'existence d'un front progressif solution de $(T F)$ si $R$ est suffisament grand, j'ai étendu la méthode développée par Emmanuel Risler dans [28] avec quelques simplifications dues au principe du maximum. L'idée est de construire le front progressif comme une limite extraite 
d'une solution de (6) pour certaines données intitiales. On montre donc l'existence d'un front, et s'il y a unicité du front limite, on a même la convergence uniforme de certaines solutions vers ce front. (6)

On appelle $u$ la solution de (6) pour une donnée initiale $u_{0}$. On définit le potentiel de l'équation

$$
F(u, z)=-\int_{0}^{u} g(v) 1_{|z| \leq R}-\alpha v 1_{|z|>R} d v
$$

Dans [6], j'ai montré le théorème suivant.

Théorème 2.4. Il existe $\delta>0$ tel qu'on ait le résultat suivant. Si $u_{0} \in H$ vérifie

$$
\limsup _{x \rightarrow+\infty} \int_{x-1}^{x+1} \int_{\mathbb{R}^{N-1}}\left(u_{0}(x, z)^{2}+\left|\nabla u_{0}(x, z)\right|^{2}\right) d z d x \leq \delta
$$

et

$$
\int_{-L}^{0} \int_{\mathbb{R}^{N-1}}\left(\frac{\left|\nabla u_{0}(x, z)\right|^{2}}{2}+F\left(u_{0}(x, z), z\right)\right) d z d x \stackrel{L \rightarrow+\infty}{\longrightarrow}-\infty,
$$

alors il existe $\tilde{x}(t)$ tel que pour toute suite $\left(t_{n}\right)_{n \in \mathbb{N}}$ avec $t_{n} \geq 0$ et $t_{n} \rightarrow+\infty$, il existe $U$ un front progressif de vitesse $c>0$ avec $U \stackrel{x \rightarrow+\infty}{\longrightarrow} 0$ et $U \stackrel{x \rightarrow-\infty}{\longrightarrow} \phi>0$, tel que, quitte à extraire,

$$
\sup _{y \in\left[-L,+\infty\left[, z \in \mathbb{R}^{N-1}\right.\right.}\left|u\left(t_{n}, \tilde{x}\left(t_{n}\right)+y, z\right)-U(y, z)\right| \stackrel{n \rightarrow+\infty}{\longrightarrow} 0
$$

pour tout $L>0$.

De plus, $\tilde{x}^{\prime}(t) \stackrel{t \rightarrow+\infty}{\longrightarrow} c$.

La démonstration de ce théorème se fait en plusieurs étapes.

Etape 1. Définition du point d'échappement.

L'idée de cette méthode est tout d'abord de supposer que la donnée initiale est proche de 0 pour $x$ proche de $+\infty$ et proche d'un état non nul plus stable que 0 pour $x$ proche de $-\infty$. C'est le sens des conditions (7) et (8) dans le théorème 2.4. On peut alors montrer que l'état non nul ne peut pas envahir l'état 0 trop rapidement [6]. Pour cela, on étudie l'évolution en $t$ et $x$ d'une énergie "locale" en $x$. Ceci permet de définir le point d'échappement $\tilde{x}(t)$ qui est le premier point où la solution $u(t, x, z)$ de (6) quitte l'état 0 du point de vue énergétique. Autrement dit, pour $x \geq \tilde{x}(t)$, $u(t, x, z)$ est proche de 0 à $\varepsilon>0$ près et pour $x<\tilde{x}(t)$, l'énergie locale de la solution $u(t, x, z)$ est supérieure ou égale à une valeur $\varepsilon>0$ bien définie.

Ces idées sont dues à Emmanuel Risler [28], bien qu'ici la définition de l'énergie de la solution soit légèrement modifiée et qu'il existe un principe du maximum.

L'idée est ensuite d'étudier l'énergie globale de la solution $u(t)$ dans un référentiel en mouvement linéaire uniforme qui "suit" le point d'échappement de façon à montrer la convergence de $u(t)$ vers une solution stationnaire non nulle dans ce référentiel. Cette solution stationnaire sera le front progressif recherché. Le premier problème est cependant de montrer que le point d'échappement a bien une vitesse asymptotique constante. Comme on va travailler dans des référentiels centrés autour du point d'échappement pour différents temps, on définit donc l'avancement maximal du point d'échappement

$$
\tilde{X}(s)=\sup _{t \geq 0}(\tilde{x}(t+s)-\tilde{x}(t)),
$$

ainsi que les vitesses asymptotiques

$$
\tilde{c}_{-}=\liminf _{t \rightarrow+\infty} \frac{\tilde{x}(t)}{t}, \quad \tilde{c}_{+}=\limsup _{t \rightarrow+\infty} \frac{\tilde{x}(t)}{t}, \quad \text { et } \quad c^{*}=\limsup _{s \rightarrow+\infty} \frac{\tilde{X}(s)}{s} .
$$


On a clairement $\tilde{c}_{-} \leq \tilde{c}_{+} \leq c^{*} \leq c_{\max }$ où $c_{\max }$ est la vitesse maximale d'envahissement de l'état 0 . Le problème est tout d'abord de montrer l'égalité de ces trois vitesses asymptotiques. Pour cela, on va étudier l'énergie de la solution dans divers référentiels en mouvement.

Etape 2. Energie dans un référentiel en mouvement.

Soit $c>0$ la vitesse du référentiel. Soit $t_{\text {init }} \geq 0$ un temps initial et $x_{i n i t}=\tilde{x}\left(t_{\text {init }}\right)$ le point d'échappement à ce temps initial.

On se place dans le référentiel en translation uniforme de vitesse $c$ dans la direction de l'axe du cylindre. Ainsi la coordonnée $z$ reste inchangée, alors que les autres coordonnées deviennent

$$
s=t-t_{\text {init }} \quad \text { et } \quad y=x-x_{\text {init }}-c s .
$$

On pose alors $v(s, y, z)=u(t, x, z)$ la solution de (6) dans ce référentiel. Elle vérifie

$$
\frac{\partial v}{\partial s}-c \frac{\partial v}{\partial y}-\triangle v+g(v) 1_{\{|z| \leq R\}}-\alpha v 1_{\{|z|>R\}}=0 .
$$

Formellement, l'énergie de la solution $v$ devrait être

$$
\Phi(s)=\iint e^{c y}\left(\frac{|\nabla v|^{2}}{2}+F(v, z)\right) d y d z
$$

et on aurait alors

$$
\Phi^{\prime}(s)=-\iint e^{c y}\left|\partial_{s} v\right|^{2} d y d z .
$$

Malheureusement, il n'y a pas de raison pour que ces termes soient bien définis, car un front d'onde est typiquement non intégrable. Il faut donc définir une nouvelle fonction de poids $\varphi(s, y)$ en tronquant l'exponentielle en un certain point $y_{0}+\gamma_{1} s$ où $\gamma_{1}$ est une constante fixée et $y_{0}$ est une constante à fixer selon le cas.

On définit donc $\varphi(s, y)$ par

$$
\varphi(s, y)= \begin{cases}e^{c y} & \text { si } y \leq y_{0}+\gamma_{1} s \\ e^{-\beta_{1} y} e^{\left(c+\beta_{1}\right)\left(y_{0}+\gamma_{1} s\right)} & \text { si } y \geq y_{0}+\gamma_{1} s\end{cases}
$$

$\beta_{1}$ étant une constante fixée.

On peut alors définir l'énergie globale de la solution

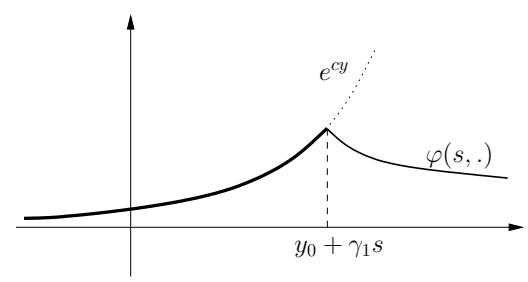

$$
\Phi(s)=\iint \varphi(s, y)\left(\frac{|\nabla v(s, y, z)|^{2}}{2}+F(v, z)\right) d y d z
$$

ainsi que son énergie de dissipation

$$
\mathcal{D}(s)=\iint \varphi(s, y) \partial_{s} v^{2}(s, y, z) d y d z
$$

Et on a la relation suivante

$$
\Phi^{\prime}(s) \leq-\frac{1}{2} \mathcal{D}(s)+K_{2} \Psi(s)
$$

où $\Psi(s)$ est un terme de pollution lié à la troncature de l'exponentielle. Quelques calculs permettent de montrer que $\mathcal{D}^{\prime}(s) \leq C \mathcal{D}(s)$ pour une certaine constante $C>0$ et que le terme de pollution $\Psi$ peut être contrôlé par $y_{0}$ sous certaines hypothèses sur la vitesse du référentiel $c>0$. Précisément, si

$$
\left(c+\beta_{1}\right)\left(c^{*}-c\right) \leq \frac{1}{4} \beta_{1} \gamma_{1},
$$


alors

$$
\int_{0}^{+\infty} \Psi(s) d s \leq \frac{1}{\varepsilon_{3}} \Psi(0)+K_{3} e^{-\beta_{1} y_{0}}
$$

avec $\varepsilon_{3}$ et $K_{3}$ des constantes fixées. Pour tous ces calculs et les définitions exactes des constantes, voir [6].

Etape 3. Vitesse asymptotique du point d'échappement.

Les estimations d'énergie précédentes vont nous permettre tout d'abord de montrer l'existence de la vitesse asymptotique du point d'échappement. Supposons par l'absurde $\tilde{c}_{-}<c^{*}$. Alors, on peut construire un référentiel de vitesse $c>$ qui vérifie

$$
\tilde{c}_{-}<c<c^{*}<c+\gamma_{1} \text { and }\left(c+\beta_{1}\right)\left(c^{*}-c\right) \leq \frac{\beta_{1} \gamma_{1}}{4}
$$

Les estimations d'énergie du paragraphe précédent sont valides dans ce référentiel, mais le point d'échappement alterne de larges excursions vers la gauche avec de larges excursions vers la droite. Ceci va contredire l'équation (10). En effet, lorsque le point fait de larges excursions vers la gauche, c'est que la solution est quasiment identiquement nulle. L'énergie globale $\Phi(s)$ est donc minorée par 0 (quitte à bien choisir le temps initial $t_{\text {init }}$ ). De l'autre coté, comme le point d'échappement fait de larges excursions vers la droite (ce qui signifie grosso modo que la solution passe de 0 à $\varepsilon$ ) et que la troncature se fait toujours après le point d'échappement ( $\operatorname{car} c^{*}<c+\gamma_{1}$ ), l'énergie de dissipation va tendre vers l'infini. Or comme le terme de pollution $\Psi$ est contrôlé par $y_{0}$, on aboutit à une contradiction avec l'estimation (10).

La vitesse asymptotique du point d'échappement sera désormais notée $\tilde{c}$.

Etape 4. Relaxation.

Maintenant que la vitesse asymptotique du point d'échappement est connue, il est possible de montrer le résultat suivant :

$$
\forall L>0 \quad\left\|\partial_{t} u(t, \tilde{x}(t)+., .)+\tilde{c} \partial_{x} u(t, \tilde{x}(t)+., .)\right\|_{L_{u l}^{2}(B(0, L))} \stackrel{t \rightarrow+\infty}{\longrightarrow} 0 .
$$

L'idée est de supposer par l'absurde que la propriété n'est pas vraie. Cela signifie que l'on peut trouver des temps initiaux $t_{n}$ aussi grand que l'on veut tels que dans le référentiel de vitesse $\tilde{c}$ et de temps initial $t_{n}$, la dissipation ne tend jamais vers 0 . Ainsi, l'énergie globale de la solution dans ces référentiels devrait tendre vers $-\infty$ d'après l'estimation d'énergie (10). Mais ces référentiels suivent de plus en plus précisément le point d'échappement, or sur $[\tilde{x}(t),+\infty[$, la solution est très proche de 0 , ce qui ne peut entraîner de large variations de l'énergie. Et sur ] $-\infty, \tilde{x}(t)$ ], la solution varie de façon limitée et comme l'énergie fait intervenir un poids exponentiel, l'énergie ne peut pas non plus tendre vers $-\infty$. Enfin, le terme de pollution peut bien être contrôlé, d'où une contradiction.

Etape 5. Conclusion sur l'existence du front.

Pour toute suite de temps $\left(t_{n}\right)_{n \in \mathbb{N}}$ avec $t_{n} \rightarrow+\infty$, on peut définir

$$
\begin{array}{cc}
w_{n}(y, z)=u\left(t_{n}, \tilde{x}\left(t_{n}\right)+y, z\right) & \text { pour }(y, z) \in \mathbb{R}^{N} . \\
\bar{w}_{n}(y, z)=\partial_{t} u\left(t_{n}, \tilde{x}\left(t_{n}\right)+y, z\right) & \text { pour }(y, z) \in \mathbb{R}^{N} .
\end{array}
$$


Comme $\left(w_{n}\right)_{n \in \mathbb{N}}$ est bornée dans l'espace de $\operatorname{Kato}^{1} \mathrm{H}_{u l}^{1}\left(\mathbb{R}^{N}\right)$ et $\left(\bar{w}_{n}\right)_{n \in \mathbb{N}}$ est bornée dans l'espace de $\operatorname{Kato}^{1} \mathrm{~L}_{u l}^{2}\left(\mathbb{R}^{N}\right)$, il existe $U \in \mathrm{H}_{u l}^{1}\left(\mathbb{R}^{N}\right)$ et $\bar{U} \in \mathrm{L}_{u l}^{2}\left(\mathbb{R}^{N}\right)$ tels que quitte à extraire une sous-suite,

$$
w_{n} \rightarrow U \text { dans } \mathrm{H}^{1}\left([-L, L]^{N}\right) \quad \text { and } \quad \bar{w}_{n} \rightarrow \bar{U} \text { dans } \mathrm{L}^{2}\left([-L, L]^{N}\right) .
$$

Alors d'après ce qui précède, $\bar{U}+\tilde{c} U=0$ et en passant à la limite dans (6), on a

$$
\triangle U+\tilde{c} \partial_{x} U-f(U, z)=0
$$

De plus, par définition du point d'échappement et de $w_{n}, U$ est non nulle, c'est donc bien un front progressif solution de (6). On peut de plus montrer la convergence uniforme sur $\left[-L,+\infty\left[\times \mathbb{R}^{N-1}\right.\right.$ pour tout $L>0$, en utilisant les propriétés de décroissance exponentielle des solutions en $+\infty$.

Enfin, pour conclure la démonstration du théorème, il reste à montrer qu'il existe bien des données initiales qui vérifient les hypothèses du théorème,

$$
\int_{-L}^{0} \int_{\mathbb{R}^{N-1}}\left(\frac{\left|\nabla u_{0}(x, z)\right|^{2}}{2}+F\left(u_{0}(x, z), z\right)\right) d z d x \stackrel{L \rightarrow+\infty}{\longrightarrow}-\infty
$$

et

$$
\limsup _{x \rightarrow+\infty} \int_{x-1}^{x+1} \int_{\mathbb{R}^{N-1}}\left(u_{0}(x, z)^{2}+\left|\nabla u_{0}(x, z)\right|^{2}\right) d z d x \leq \delta .
$$

Pour cela, il suffit de trouver un profil asymptotique de l'équation tel que

$$
E(\phi)=\int_{\mathbb{R}^{N-1}}\left(\frac{|\nabla \phi|^{2}}{2}+F(\phi, z)\right) d z<0 .
$$

En effet, dans ce cas,

$$
u_{0}(x, z)= \begin{cases}\phi(z) & \text { si } x \leq 0 \\ \phi(z) e^{-x} & \text { si } x>0\end{cases}
$$

convient comme donnée initiale.

Ce résultat se démontre en remarquant tout d'abord que

$$
E(\phi)=\int_{\mathbb{R}^{N-1}}\left(F(\phi, z)-\frac{f(\phi, z)}{2} \phi\right) d z
$$

où $f(\phi, z)=g(\phi) 1_{\{|z| \leq R\}}-\alpha \phi 1_{\{|z|>R\}}$. Pour $|z|>R, F(\phi, z)-\frac{f(\phi, z)}{2} \phi=0$, il reste donc a étudier

$$
F(\phi, z)-\frac{f(\phi, z)}{2} \phi=-G(\phi)+\frac{g(\phi)}{2} \phi
$$

pour $z \in B(0, R)$ ( $G$ est la primitive de $g$ qui s'annule en 0$)$. En dimension 2, en utilisant le plan de phase, il n'est pas très compliqué de montrer que la zone où $-G(\phi)+\frac{g(\phi)}{2} \phi<-\varepsilon$ croit infiniment avec $R$, alors que la zone où $-G(\phi)+\frac{g(\phi)}{2} \phi \geq-\varepsilon$ reste bornée. Cela montre que si $R$ est suffisament grand, la solution $\phi_{2}$ en dimension 2 est bien d'énergie négative. Pour la dimension supérieure, la solution en dimension 2 étant une sous-solution, la conclusion est la même.

\footnotetext{
${ }^{1}$ On définit les espaces de Kato par

$$
\mathrm{H}_{\mathrm{ul}}^{k}\left(\mathbb{R}^{N}\right)=\left\{u \in \mathrm{H}_{\mathrm{loc}}^{k}\left(\mathbb{R}^{N}\right) \mid\|u\|_{\mathrm{H}_{\mathrm{ul}}^{k}}<+\infty, \lim _{X \rightarrow 0}\left\|T_{X} u-u\right\|_{\mathrm{H}_{\mathrm{ul}}^{k}}=0\right\}
$$

où $T_{X} u(Y)=u(Y-X)$ et $\|u\|_{\mathrm{H}_{\mathrm{ul}}^{k}}=\sup _{X \in \mathbb{R}^{N}}\left\|u_{\mid B(X, 1)}\right\|_{\mathrm{H}^{k}(B(X, 1))}$.
} 


\section{Conclusion et perspectives}

La modélisation des DCE a donc permis l'élaboration de nouvelles hypothèses biologiques pour expliquer les difficultés d'observation de ces ondes dans le cerveau humain, mais elle a aussi soulevé de nouvelles questions mathématiques sur l'existence de fronts progressifs dans diverses géométries. Ce modèle de DCE a par la suite été introduit dans un modèle global d'AVC [7]. Les résultats obtenus sur le rôle des DCE dans l'aggravation des dommages suite à la reperfusion des AVC sont intéressants (en cours de publication). Ce modèle pourrait également être utilisé pour étudier d'autres pathologies (migraine avec aura ou epilépsie). Enfin, les effets d'une géométrie réaliste de cerveau (coupe de cerveau 2D ou cerveau 3D) sont en cours d'étude.

Au niveau mathématique, de nombreuses questions restent posées. Quel est l'effet d'un élargissement progressif de la matière grise? L'analyse numérique a déjà donné quelques résultats, mais aucune démonstration théorique n'est disponible à ce jour. L'effet de la courbure de la matière grise en présence de matière blanche semble également très intéressant. Selon que la matière blanche est à l'intérieur ou à l'extérieur de la courbe formé par la matière grise, le front va être accéléré ou ralenti. Là aussi, tout reste à faire. Enfin, il est bien connu que des neurones y compris très éloignés peuvent être reliés entre eux par des synapses. La dépolarisation d'un neurone entraine alors un début de dépolarisation de tous les neurones qui lui sont connectés. Ainsi, les DCE ne seraient pas simplement créées par un mécanisme de réaction-diffusion locale, il faudrait y ajouter un terme de diffusion non locale (ajout d'un terme intégral dans le système). L'influence de ce terme sur la vitesse des ondes pourrait être intéressant à étudier.

\section{RÉFÉRENCES}

[1] Aitken, P.G.; Jing, J. ; Young, J. ; Friedman, A.; Somjen, G.G. Spreading depression in human hippocampal tissue in vitro. Third IBRO Congr. Montreal Abstr., 1991, 329.

[2] Aronson, D.G.; Weinberger, H.F. Nonlinear diffusion in population genetics, combustion, and nerve pulse propagation. Partial differ. Equat. relat. Top. 1975, Lect. Notes Math. 446, 5-49.

[3] Berestycki, H. ; Nirenberg, L. Travelling fronts in cylinders. Ann Inst Henri Poincaré 1992, Anal. Non linéaire 9 (5), 497-572.

[4] Berestycki, H.; Nirenberg, L. Monotonicity, symmetry and antisymmetry of solutions of semilinear elliptic equations. [J] J. Geom. Phys. 1988 5, No.2, 237-275.

[5] Chan, P.H. Cerebrovascular Disease 22nd Princeton Conference. Cambridge University Press, 2002.

[6] Chapuisat, G. Existence and non-existence of curved front solution of a biological equation. Journal of Differential Equations 2007 236, No.1, 237-279

[7] Chapuisat, G.; Grenier, E.; Dronne, M.A.; Hommel, M.; Boissel, J.P. A global model of ischemic stroke with stress on spreading depression. Progress in Biophysics and Molecular Biology 200897, 4-27

[8] Chapuisat, G. Discussion of a simple model of spreading depression. 2007 ESAIM Proc 18, 87-98.

[9] Chapuisat, G.; Grenier, E. Existence and nonexistence of traveling wave solutions for a bistable reactiondiffusion equation in an infinite cylinder whose diameter is suddenly increased. Commun. Partial Differ. Equations (2005) 30, No.10-12, 1805-1816

[10] Evans, L.C. Partial differential equations. Graduate Studies in Mathematics. 19. Providence, RI : American Mathematical Society (AMS). xvii, 662 p. 1998.

[11] Fife, P.C.; Mac Leod, J.B. The approach of solutions of nonlinear diffusion equations to travelling front solutions. Arch Ration Mech Anal 1977, 65, 335-361.

[12] Fife, P.C.; Mac Leod, J.B. A phase plane discussion of convergence to travelling fronts for nonlinear diffusion. Arch Ration Mech Anal 1981, 75, 281-314.

[13] Gardner, R.A. Topological methods for the study of travelling wave solutions of reaction diffusion systems. Reaction-diffusion equations 1990, Proc. Symp. Year, Edinburgh/UK 1987-1988, 173-198.

[14] Grafstein, B. Neuronal release of potassium during spreading depression. In M.A.B. Brazier (eds), Brain function : Cortical Excitability and Steady Potentials. University of California Press : Berkeley, 1963.

[15] Hadjikhani, N.; Sanchez del Rio, M.; Wu, O.; Schwartz, D.; Bakker, D.; Fischl, B.; Kwong, K.K.; Cutrer, F.M.; Rosen, B.R.; Tootell, R.B.; Sorensen, A.G., Moskowitz, M.A. Mechanism of migraine aura revealed by functional MRI in human visual cortex. Proc. Natl. Acad. Sci. USA 2001, 98, 4687-4692.

[16] Hamel, F.; Monneau, R.; Roquejoffre, J.M. Existence and qualitative properties of multidimensional conical bistable fronts. Discrete Contin. Dyn. Syst. 2005 13, No.4, 1069-1096. 
[17] James, M.F.; Smith, J.M.; Boniface, S.J.; Huang, C.L.; Leslie, R.A. Cortical spreading depression and migraine : new insights from imaging?. Trends Neurosci 2001, 24 (5) 266-271.

[18] Leão, A.A.P. Spreading depression of activity in the cerebral cortex. J Neurophysiol, 1944, 10 (7), 359-390.

[19] Mayevsky, A.; Doron, A.; Manor, T.; Meilin, S. ; Zarchin, N.; Ouaknine, G.E. Cortical spreading depression recorded from the human brain using a multiparmetric monitoring system. Brain Res. 1996, 740, 268-274.

[20] McLachlan, R.S.; Girvin, J.P. Spreading depression of Leao in rodent and human cortex. Brain Res. 1994, 666(1), 133-136.

[21] Mies, G.; Iijima, T.; Hossman, K.A. Correlation between peri-infarct DC shifts and ischaemic neuronal damage in rat. Neuroreport, 1993, 4, 709-711.

[22] Nicholson, C. Volume transmission and the propagation of spreading depression. In A. Lehmenkühler, K.-H. Grotemeyer and F. Tegtmeier (eds), Migraine : basic mechanisms and treatment. Urban \& Scwarzenberg : München, 293-308, 1993.

[23] Obeidat, A.S.; Jarvis, C.R.; Andrew, R.D. Glutamate does not mediate acute neuronal damage after spreading depression induced by $\mathrm{O} 2$ /glucose deprivation in the hippocampal slice. J Cereb Blood Flow Metab 2000, 20 (2), 412-422.

[24] Protter, M.H.; Weinberger, H.F. Maximum principles in differential equations. Prentice hall : Englewood Cliffs, 1967.

[25] Rash, J.E.; Yasumura, T.; Dudek, F.E.; Nagy, J.I. Cell-specific expression of connexins and evidence of restricted gap junctional coupling between glial cells and between neurons. J Neurosci 2001, 21 (6), 1983-2000.

[26] Reggia, J.; Montgomery, D. A computational model of visual hallucinations in migraine. Comput Biol Med 1996, 26 (2), 133-141.

[27] Revett, K.; Ruppin, E.; Goodall, S.; Reggia, J.A. Spreading depression in focal ischemia : a computational study. J Cereb Blood Flow Metab 1998, 18 (9), 998-1007.

[28] Risler, E. Global convergence toward traveling fronts in nonlinear parabolic systems with a gradient structure, Annales IHP(C) Non Linear Analysis 2008, 25 (2),381-424.

[29] Roquejoffre, J.-M. Eventual monotonicity and convergence to travelling fronts for the solutions of parabolic equations in cylinders. Ann Inst Henri Poincaré 1997, Anal. Non linéaire 14 (4), 499-552.

[30] Shapiro, B. Osmotic forces and gap junctions in spreading depression : a computational model. J Comput Neurosci 2001, 10 (1), 99-120.

[31] Smoller, J. Shock waves and reaction-diffusion equations, 2nd Ed.; Grundlehren der Mathematischen Wissenschaften ; Springer-Verlag :New-York, 1994; 258p.

[32] Somjen, G. Ions in the Brain : Normal Function, Seizures, and Stroke. Oxford University Press : New York, 2004.

[33] Šramka, M.; Brozek, G.; Buresš, J.; Nádvorník, P. Functional ablation by spreading depression : possible use in human stereptactic surgery. Appl. Neurophysiol. 1977, 40, 48-61.

[34] Tuckwell, H.C. ; Predictions and properties of a model of potassium and calcium ion movements during spreading cortical depression. Int J Neurosci 1980, 10 (2-3), 145-164.

[35] Vega, J.M. The asymptotic behavior of the solutions of some semilinear elliptic equations in cylindrical domains. J. Differ. Equations 1993 102, No.1, 119-152.

[36] Volpert, V.A.; Volpert, A.I. Multidimensional travelling waves in the bistable case. Amann, H. (ed.) et al., Progress in partial differential equations. Papers from the 3rd European conference on elliptic and parabolic problems, Pont-à-Mousson, France, June 1997. Vol. 2. Harlow : Longman. Pitman Res. Notes Math. Ser. 384, 156-173, 1998. 